\title{
LEVANTAMENTO DA COMPOSIÇÃO FLORÍSTICA DE UMA FLORESTA ESTACIONAL SEMIDECIDUAL LOCALIZADA NO MUNICÍPIO DE CÁSSIA-MG
}

\author{
Márcio Coraiola ${ }^{1}$; Sylvio Péllico Netto ${ }^{2}$
}

\section{Resumo}

O presente trabalho de pesquisa teve como principal objetivo o levantamento da composição florística de uma Floresta Estacional Semidecidual. Para avaliação da composição florística da floresta foram considerados os indivíduos com DAP $\geq 10 \mathrm{~cm}$, observados em 12 unidades amostrais de 1 hectare $(100 \mathrm{~m} \times 100 \mathrm{~m})$ distribuídas sistematicamente na área. A regeneração natural da floresta foi amostrada por meio da metodologia de Strand, com 48 unidades amostrais distribuídas aleatoriamente na população, nas quais foram levantados os indivíduos com DAP $<10 \mathrm{~cm}$. A floresta estudada apresenta uma composição florística heterogênea, com 124 espécies, distribuídas em 99 gêneros e 46 famílias botânicas. No caso da regeneração natural, foram encontradas apenas 69 espécies arbóreas (DAP menor que $10 \mathrm{~cm}$ ), distribuídas em 58 gêneros e 33 famílias botânicas

Palavras-chave: Análise estrutural, Composição florística, Regeneração natural, Floresta natural.

\section{Abstract}

The present research paper had as main objective, the survey of floristic composition of a "Semidecidual Tropical Florest". For the evaluation of floristic composition were used 12 sampling units (100m x 100m), were used systematically distributed over the area, where all the trees with dbh larger or equal than $10 \mathrm{~cm}$ were measured. The natural regeneration was sampled using Strand's Sampling Method, with 48 sampling units distributed in the population at random, where all individuals with dbh smaller than $10 \mathrm{~cm}$ were measured. The studied forest area presented a heterogenic floristic composition, with 124 species distributed into 99 genera and 46 botanic families. The natural regeneration, whose floristic composition differ in relation to the forest, presented just 69 species distributed into 58 genera and 33 botanic families.

Keywords: Structure analysis, Floristic composition, Natural regeneration, Tropical forest.

1 Eng. Florestal, M. Sc., Doutorando do curso de Pós-Graduação em Engenharia Florestal da UFPR e Professor Assistente do CCAA/PUCPR

2 Eng. Florestal, M. Sc., Dr., Professor adjunto do CCAA/PUCPR, Bolsista do CNPq.

BR 376 km 14, Costeira, CEP 83.010-500, São José dos Pinhais, PR

Telefones: (41) 382-1454, Fax: (41) 382-1223

pelico@rla13.pucpr.br 


\section{Introdução}

As florestas naturais constituem-se em um ecossistema extremamente complexo, cujo equilíbrio pode ser facilmente alterado caso haja perturbações expressivas no meio. Entretanto, estas florestas não devem ser consideradas como um ecossistema intocável, e sim manejada de maneira racional, visando a proteção e manutenção das suas características naturais (conservação dos solos, regulação do regime hídrico, estabilização climática, preservação da flora e fauna etc.)

O conhecimento da composição florística da floresta e da regeneração natural, aliados a outros levantamentos estruturais, se constituem nos aspectos mais importantes para a implantação de qualquer plano de manejo destes recursos. Com base na identificação científica das espécies, pode-se dar início às análises da estrutura horizontal, vertical e dimensional, essenciais para o conhecimento da dinâmica da floresta.

Baseado nesta questão, pretende-se, com o presente trabalho, efetuar a descrição da composição florística de uma Floresta Estacional Semidecidual, localizada no município de CássiaMG, destacando os indivíduos da floresta e da regeneração natural.

Para HUSCH et al. (1972), a estrutura do povoamento pode ser definida como a distribuição de espécies e quantidade de árvores numa área florestal, sendo o resultado dos hábitos de crescimento das espécies e das condições ambientais onde o povoamento se originou e desenvolveu.

Mesmo não havendo uma metodologia padrão para analisar as comunidades florestais, que são basicamente produtos da diferença de tolerância das espécies em relação ao meio (amplitude ecológica) e da heterogeneidade do meio, qualquer procedimento adota os seguintes requisitos (LAMPRECHT, 1964): ser capaz de dar uma visão representativa da estrutura da floresta estudada, ser aplicável a qualquer tipo de comunidade florestal, que os resultados sejam livres de influências subjetivas, que os resultados de diferentes análises ou de diferentes comunidades florestais sejam passíveis de comparações entre si, e que sejam aplicáveis aos métodos estatísticos modernos na compilação e comparação dos resultados.
Para HOSOKAWA (1986), as florestas naturais possuem elevada diversidade de espécies e uma grande variação de qualidades em termos econômicos. Assim, os levantamentos estruturais deverão abranger pelo menos os seguintes itens: estrutura horizontal, estrutura vertical, estrutura paramétrica e estrutura da regeneração natural.

\section{Material e métodos Área de Estudo}

Os dados básicos utilizados neste trabalho foram obtidos em inventário florestal realizado numa área de floresta natural, localizada no município de Cássia, região sul do Estado de Minas Gerais, situada entre: Latitude 20œ20' e $20 \infty 40$ ' Sul e Longitude 46040' e 47000' Oeste (FIGURA 1). A área de estudo é formada por uma propriedade denominada como Fazenda Reata, possuindo cerca de 90 hectares de floresta natural.

FIGURA 1 - LOCALIZAÇÃO DA CIDADE DE CÁSSIA NO ESTADO DE MINAS GERAIS - BRASIL

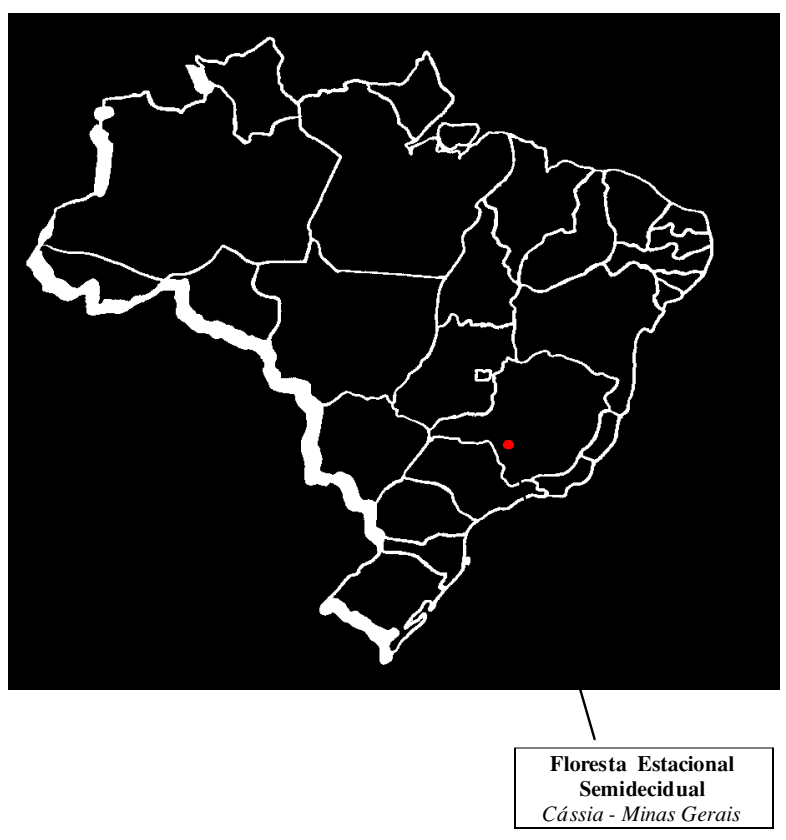


A região de Cássia-MG está situada a, aproximadamente, 680 metros de altitude, e apresenta temperaturas médias anuais de 26,500C (máxima) e $19,5 \infty \mathrm{C}$ (mínima). O clima da região, segundo classificação de KÖppen, é do tipo Cwa (Tropical de altitude), apresentando verões rigorosos e chuvosos.

A vegetação predominante na região estudada é a Floresta Estacional Semidecidual. O conceito ecológico da região da Floresta Estacional está preso ao clima de duas estações, uma chuvosa e outra seca, que condicionam uma estacionalidade foliar dos elementos arbóreos dominantes, os quais têm adaptação fisiológica à deficiência hídrica ou à baixa temperatura, durante certo tempo. No caso das Florestas Semideciduais, a porcentagem de árvores caducifólias no conjunto florestal, e não das espécies que perdem folhas individualmente, deve-se situar em torno de 20 a $50 \%$ na época desfavorável (RADAM-BRASIL, 1978).

A formação encontrada na área de estudo compreende a Floresta Montana, que apresenta o maior número de agrupamentos remanescentes na área abrangida pelos ambientes da Floresta Estacional Semidecidual. Ela ocorre nas altitudes entre 500 e 1500 metros, revestindo os diques de diabásio da Formação Serra Geral, na bacia do Paraná, e sobre o relevo dissecado do embasamento de litologia variada. Apesar do número elevado de agrupamentos remanescentes, eles são na sua maioria pequenos, sendo os mais representativos encontrados próximos às cidades de Cordeiro-MG, Trajano de Moraes-RJ, Dores de Turvo-MG, Alvinópolis-MG, Carmo do Meio-MG e Bocaína de Minas-MG. Nestas áreas são freqüentes espécies dos gêneros Aspidosperma (perobas), Piptadenia (angicos), Cariniana (jequetibás), Ocotea e Nectandra (ca- nelas), e Lecythis (sapucáia), que ocupam o estrato dominante da floresta (RADAM-BRASIL, 1978).

\section{Metodologia}

Para avaliação da composição florística da floresta, foram utilizadas 12 unidades amostrais (100 x 100 metros), nas quais se considerou todos os indivíduos com DAP maior ou igual a $10 \mathrm{~cm}$. Para a levantamento da regeneração natural foram levantados todos os indivíduos arbóreos com DAP menor que $10 \mathrm{~cm}$, considerando para tal, 4 unidades amostrais instaladas em cada uma das unidades anteriores, resultando num total de 48 unidades para a floresta.

A unidade de Strand é constituída de uma linha de 15,78 metros de comprimento instalada aleatoriamente na floresta, na qual se classifica a regeneração baseada em um critério proporcional à altura dos indivíduos (PÉLLICO NETTO et al., 1997).

A identificação das espécies por meio do material botânico coletado foi realizada no Laboratório de Dendrologia do Curso de Engenharia Florestal da UFPR e no Museu Botânico Municipal de Curitiba.

\section{Resultados e discussão Composição florística}

Na TABELA 1 estão apresentadas todas as espécies que ocorreram na área estudada com DAP $>10 \mathrm{~cm}$, com identificação dos nomes vulgares e científicos, bem como os números das parcelas em que ocorreram.

\begin{tabular}{|c|c|c|c|}
\hline \multicolumn{4}{|c|}{ TABELA 1 - COMPOS IÇÃO FLORÍSTICA DA FLORESTA } \\
\hline CÓDIGO & NOME VULGAR & NOME CIENTÍFICO & FAMÍLIA \\
\hline 1001 & Açoita cavalo & Cordia sp. 2 & Boraginaceae \\
\hline 1002 & Alecrim & Holocalix balanseae Micheli & Mimosaceae \\
\hline 1003 & Amenduim & Sennasp.1 & Caesalpinaceaea \\
\hline 1004 & Amesca & Trichilia pallida $\mathrm{Sw}$. & Meliaceae \\
\hline 1005 & Amoreira & Maclura trinctoria (L.) Don ex Ste udel & Moraceae \\
\hline 1006 & Angá graúdo & Inga sp. & Mimosaceae \\
\hline
\end{tabular}




\begin{tabular}{|c|c|c|c|}
\hline CODIGO & NOME VULGAR & NOME CIENTIFICO & FAMILIA \\
\hline 1007 & Angá III & Fabaceae 1 & Fabaceae \\
\hline 1008 & Angá miúdo & Inga marginata Wild. & Mimos aceae \\
\hline 1010 & Ariticum cagão & Annona cacans Warms. & Anonaceae \\
\hline 1011 & Ariticunzinho & Annona montana Macfad; R. F. Fries & Annonaceae \\
\hline 1013 & Arruda & Zanthoxylum rhoifolium Lam. & Rutaceae \\
\hline 1014 & Bálsamo & Myrocarpus frondosus Fr. Allen. & Fabaceae \\
\hline 1015 & Batalha & Lauraceae 2 & Lauraceae \\
\hline 1016 & Bico de pato & Machaerium aculeatum Raddi & Fabaceae \\
\hline 1017 & Braúna & Schinopsis brasiliensis Engl. & Anacardiaceae \\
\hline 1018 & Cambará lixa & Aloysia virginata Juss. & Verbenaceae \\
\hline 1019 & Camboatá & Cupania vernalis $\mathrm{Camb}$. & Sapindaceae \\
\hline 1020 & Cambuí & Myrtaceae 4 & Myrtaceae \\
\hline 1021 & Canafístula & Cassia ferruginea Schrad. ex DC. & Caesalpinaceae \\
\hline 1022 & Canela & Lauraceae 5 & Lauraceae \\
\hline 1023 & Canela amarela & Nectandra sp. & Lauraceae \\
\hline 1024 & Canela bosta & Nectandra megapotamica Mez. & Lauraceae \\
\hline 1025 & Canela branca & Lauraceae 4 & Lauraceae \\
\hline 1026 & Canela preta & Ocotea $s p$ & Lauraceae \\
\hline 1027 & Canela sebo & Lauraceae 1 & Lauraceae \\
\hline 1028 & Canjerana & Cabralea canjerana (Vell.) Martius. & Meliaceae \\
\hline 1029 & Canjica & Mimosaceae 1 & Mimos aceae \\
\hline 1031 & Capitão & Terminaliasp. 2 & Combretaceae \\
\hline 1032 & Capixingui & Croton floribundus Spreng. & Euphorbiaceae \\
\hline 1033 & Capororocão & Myrsine umbellata Mez. & Myrsinaceae \\
\hline 1034 & Carne de vaca & Roupala sp. & Proteaceae \\
\hline 1035 & Caroba & Jacaranda cf. micrantha CHAM. & Bignoniaceae \\
\hline 1036 & Casca de arroz & Myrciasp. & Myrtaceae \\
\hline 1037 & Cedro & Cedrella cf. fissilis Vell. & Meliaceae \\
\hline 1038 & Coquinho catarro & Arecaceae 1 & Arecaceae \\
\hline 1039 & $\mathrm{D}$ & Desconhecida & Desconhecida \\
\hline 1040 & D1 & Trichill ia clausennii & Meliaceae \\
\hline 1041 & D4 & Siparuna apiosyce (Mart.) DC. & Monimiac eae \\
\hline 1042 & Embaúba & Cecropia pachysta chya Trec. & Cecropiaseae \\
\hline 1043 & Erva de lagarto & Casearia sylvestris Sw. & Flacourtiaceae \\
\hline 1045 & Farinha seca & Albizia polycephalla (Benth) Killip. & Mimosaceae \\
\hline 1046 & Figueira & Ficus sp. 1 & Moraceae \\
\hline 1048 & Fruteira & Eugenia sp. & Myrtaceae \\
\hline 1050 & Gairova & Syagrus oleraceae (Mart.) Becc. & Arecaceae \\
\hline 1051 & Gameleiro & Ficus sp. 2 & Moraceae \\
\hline 1052 & Guaritá & Astronium graveolens Jacq. & Anacardiaceae \\
\hline 1053 & Guatambú café & Aspidosperma ramiflorum M. Arg. & Apocynaceae \\
\hline 1054 & Imbira sapo & Lonchocarpus sp. & Fabac eae \\
\hline 1055 & Ipê amarelo & Tabebuia sp. 2 & Bignoniaceae \\
\hline 1057 & Jacarandá roxo & Machaerium sp. & Fabac eae \\
\hline 1058 & Jambreiro & Clusiaceae 1 & Clusiaceae \\
\hline 1059 & Jaracatiá & Jaracatia spinosa (Aubl) A.DC. & Carycaceae \\
\hline
\end{tabular}




\begin{tabular}{|c|c|c|c|}
\hline CÓDIGO & NOME VULGAR & NOME CIENTÍFICO & FAMÍLIA \\
\hline 1060 & Jatobá & Hymenaea courbaril Linn. & Caesalpinaceae \\
\hline 1061 & Jequetibá branco & Carinia na estrellensis (Mart.) Kuntze & Lecytidaceae \\
\hline 1062 & Jequetibá rosa & Carinia na legalis (Raddi) Kuntze & Lecytidaceae \\
\hline 1064 & Limeira & Citrus sp. & Rutaceae \\
\hline 1065 & Mamica de porca & Zanthoxylum sp. & Rutaceae \\
\hline 1066 & Mandiocão & Shefflera sp. & Araliaceae \\
\hline 1067 & Marinheirinho & Matayba elaegnoides Radl k. & Sapindaceae \\
\hline 1068 & Marinheiro & Guarea Kunthiana Juss. & Meliaceae \\
\hline 1069 & Monjoleiro & Acacia polyphylla DC. & Mimosaceae \\
\hline 1071 & Óleo de copaíba & Copaifera langsdorffii Desf. & Caesalpinaceae \\
\hline 1072 & Orelha de mateiro & Chrysophyllum gonocarpum (Mart \& Eichl) Engl. & Sapotaceae \\
\hline 1073 & Orvalho & Trichillia pallens $\mathrm{C}$. & Meliaceae \\
\hline 1074 & Paineira & Chorisia speciosa St. Hill. & Bombacaceae \\
\hline 1075 & Paineira branca & Pseudobombax grandiflorum (Carv.) A. Robins & Bombacaceae \\
\hline 1076 & Palmito & Euterpe edul is Mart. & Arecaceae \\
\hline 1077 & Panacéia & Solanum cernuum Vell. & Solanaceae \\
\hline 1079 & Pau alho & Galesia integrifolia Spreng. Harms. & Phytollacaceaea \\
\hline 1081 & Pau pólvora & Trema micrantha Blume. & Ulmaceae \\
\hline 1082 & Pau terra & Centrolobium $s p$. & Fabaceae \\
\hline 1083 & Pau viola & Alchornea triplinervia Muell. Arg. & Euphorbiaceae \\
\hline 1084 & Pereira & Platyciamus regnelli Benth. & Fabaceae \\
\hline 1085 & Peroba & Aspidosperma sp. 4 & Apocynaceae \\
\hline 1086 & Peroba branca & Aspidosperma sp. 3 & Apocynaceae \\
\hline 1087 & Peroba canela de velha & Aspidosperma sp. 2 & Apocynaceae \\
\hline 1088 & Peroba poca & Aspidosperma sp. 1 & A pocynaceae \\
\hline 1089 & Peroba rosa & Aspidosperma polyneuron Muell. Arg. & Apocynaceae \\
\hline 1090 & Peroba vermelha & Aspidosperma pyricollum $\mathrm{M}$ art. & A pocynaceae \\
\hline 1091 & Pessegueiro bravo & Prunus subcoriaceae Koehne. & Rosaceae \\
\hline 1092 & Pindaíba & Xylopia sp. & Annonaceae \\
\hline 1095 & Quaresma & Miconia discolor DC. & Melastomataceae \\
\hline 1096 & Quatiguá & Trichillia $s p$. & Meliaceae \\
\hline 1098 & Sangueiro & Pterocarpus violaceous Vog. C19 & Fabaceae \\
\hline 1099 & Sassafrás & Ocotea pretiosa Benth. \& Hook. & Lauraceae \\
\hline 1100 & Serralha & Soracea guille rminiania Gaudich. & Moraceae \\
\hline 1101 & Se te casaco & Myrta ceae 1 & Myrtaceae \\
\hline 1102 & Tamburilo & Enterolobium contorstisiliquum Morong. & Mimosaceae \\
\hline 1103 & Tento & Ormosia arborea Harnu. & Fabaceae \\
\hline 1104 & Três folhas & Esenbeckia grandiflora Mart. & Rutaceae \\
\hline 1105 & Unha de boi & Bauhinia fortificata Link. & Mimosaceae \\
\hline 1106 & Urtigão & Urera baccifera Gaudich. & Urticaceae \\
\hline 1108 & Veludo & Chomesia sp. & Rubiaceae \\
\hline 1109 & Vinhático & Vochysia tucanorum Mart. & Vochysiaceae \\
\hline 1110 & Allophyllus & Allophyllus sericeus (Camb.) Radlk. & Sapindace ae \\
\hline 1111 & Buchanaria & Buchanaria sp. & Combretac eae \\
\hline 1112 & Mangue & Calophyllum brasiliense Camb. & Clusiaceae \\
\hline 1114 & Casearia & Casearia $s p$. & Flac ourtiaceae \\
\hline
\end{tabular}




\begin{tabular}{|c|c|c|c|}
\hline CÓDIGO & NOME VULGAR & NOME CIENTÍFICO & FAMÍLIA \\
\hline 1115 & Sobraji & Columbrina glandulosa Perkins & Rhamnaceae \\
\hline 1116 & Cordia 1 & Cordiasp. 1 & Boraginaceae \\
\hline 1117 & Cordia 2 & Cordiasp. 3 & Boraginaceae \\
\hline 1118 & Maria-mole & Dendropanax cuneatum (DC) Pland. & Araliaceae \\
\hline 1119 & Hesteria & Hesteria sp. & Ola caceae \\
\hline 1121 & Myroloxum & Myroloxum sp. & Fabac eae \\
\hline 1122 & Nyctaginaceae 1 & Nyctaginaceae 1. & Nyctaginaceae \\
\hline 1123 & Almecegueira & Protium Heptaphyllum (Aubl.) Mach. & Burseraceae \\
\hline 1124 & Psychotria & Psychotria cf. mapourioides DC. & Rubiaceae \\
\hline 1125 & Rollinia & Rollinia sp. & Annonaceae \\
\hline 1126 & Sloaneae & Sloanea guianiensis (Aubl.) Bentham. & El aeocarpaceae \\
\hline 1127 & Sola num & Solanum schuartzianum R\& S. & Solaneceae \\
\hline 1128 & Styrax 1 & Styrax sp. 1 & Styracaceae \\
\hline 1129 & Bombacapsis & Bombacopsis sp. & Bombacaceae \\
\hline 1131 & Hirtella & Hirtella $s p$ & Chrysobalanaceae \\
\hline 1132 & Lauraceae 3 & Lauraceae 3 & Lauraceae \\
\hline 1134 & Myrtac eae 5 & Myrtac eae 5 & Myrtaceae \\
\hline 1136 & Rubiaceae 1 & Rubiaceae 1 & Rubiaceae \\
\hline 1137 & Rubiaceae 2 & Rubiaceae 2 & Rubiaceae \\
\hline 1139 & Styrax 2 & Styrax sp. 2 & Styracaceae \\
\hline 1140 & Ipê felpudo & Tabebuia sp. 1 & Bignoniaceae \\
\hline 1141 & Tocayena & Tocayena sp. & Rubiaceae \\
\hline 1142 & Virola & Virolasp. & Miristicaceae \\
\hline 1143 & Pindaubuna & Xylopia brasiliensis Spreng. & Annonaceae \\
\hline 1145 & Terminalia 1 & Terminalia sp. 1 & Combretaceae \\
\hline
\end{tabular}

A Tabela 2 apresenta a composição florística encontrada na regeneração natural da floresta, contendo todos os indivíduos com DAP $<10 \mathrm{~cm}$, com identificação dos nomes vulgares e científicos, e o número total de parcelas em que ocorreram.

\begin{tabular}{|c|c|c|c|c|}
\hline CÓDIGO & NOME VULGAR & NOME CIENTÍ FICO & FAMÍLIA & $\begin{array}{c}\text { OCORRÊNCIA } \\
\text { (N }^{\circ} \text {. DE } \\
\text { PARCELAS) }\end{array}$ \\
\hline 1002 & Alecrim & Holocalix balansae Micheli & Mimosaceae & 1 \\
\hline 1004 & Almescla & Trichilia pallida $\mathrm{Sw}$. & Meliaceae & 33 \\
\hline 1006 & Ingágraúdo & Inga sp. & Mimosaceae & 14 \\
\hline 1007 & IngáIII & Fabaceae 1 & Fabaceae & 2 \\
\hline 1008 & Ingámiúdo & Inga marginata $\mathrm{W}$ ild. & Mimosaceae & 18 \\
\hline 1011 & Araticunzinho & Annona montana Macfad; R. F. Fries & Annonaceae & 10 \\
\hline 1013 & Arruda & Zanthoxylum rhoifolium Lam. & Rutaceae & 1 \\
\hline 1014 & Bálsamo & Myrocarpus frondosus Fr. Allen. & Fabaceae & 7 \\
\hline 1015 & Batalha & Lauraceae 2 & Lauraceae & 3 \\
\hline 1016 & Bico de pato & Machaerium aculeatum Raddi & Fabaceae & 4 \\
\hline 1017 & Braúna & Schinopsis brasiliensis Engl. & Anacardiaceae & 1 \\
\hline 1019 & Camboatá & Cupania vernalis Camb. & Sapindaceae & 8 \\
\hline 1020 & Cambuí & Myrtaceae 4 & Myrtaceae & 8 \\
\hline 1023 & Canela amarela & Nectandra sp. & Lauraceae & 22 \\
\hline
\end{tabular}




\begin{tabular}{|c|c|c|c|c|}
\hline CÓDIGO & NOME VULGAR & NOME CIENTÍFICO & FAMÍLIA & $\begin{array}{c}\text { OCORRENCIA } \\
\left(N^{\circ} . \text { DE }\right. \\
\text { PARCELAS) }\end{array}$ \\
\hline 1027 & Canela sebo & Lauraceae 1 & Lauraceae & 2 \\
\hline 1028 & Canjerana & Cabralea canjerana (Vell.) Martius. & Meliaceae & 3 \\
\hline 1029 & Canjica & Mimosaceae 1 & Mimosaceae & 7 \\
\hline 1031 & Capitão & Terminalia sp. 2 & Combretaceae & 4 \\
\hline 1032 & Capixingui & Croton floribundus Spreng. & Euphorbiaceae & 20 \\
\hline 1033 & Capororocão & Myrsine umbellata Mez. & Myrsinace ae & 11 \\
\hline 1034 & Carne de vaca & Roupala sp. & Proteaceae & 6 \\
\hline 1035 & Caroba & Jacaranda cf. micrantha CHAM. & Bignoniaceae & 1 \\
\hline 1036 & Casca de arroz & Myrcia sp. & Myrtaceae & 1 \\
\hline 1039 & $\mathrm{D}$ & Desconhecida & Desconhecida & 42 \\
\hline 1040 & D1 & Trichillia claus ennii & Meliaceae & 31 \\
\hline 1041 & D4 & Siparuna apiosyce (Mart) DC. & Monimiaceae & 11 \\
\hline 1042 & Embaúba & Cecropia pachystachya Trec. & Cecropiaceae & 1 \\
\hline 1043 & Erva de lagarto & Casearia sylvestris Sw. & Flacourtiaceae & 2 \\
\hline 1045 & Farinha seca & Albizia polycephalla (Benth) Killip. & Mimosaceae & 7 \\
\hline 1050 & Gairova & Syagrus oleraceae (Mart.) Becc. & Arecaceae & 1 \\
\hline 1052 & Guaritá & Astronium graveolens Jacq. & Anacardiaceae & 33 \\
\hline 1053 & Guatambú café & Aspidosperma ramiflorum M. Arg. & Apocynaceae & 7 \\
\hline 1055 & Ipê amarelo & Tabebuia sp. 2 & Bignoniaceae & 2 \\
\hline 1058 & Jambreiro & Clusiaceae 1 & Clusiaceae & 24 \\
\hline 1059 & Jaracatiá & Jaracatia spinosa (Aubl) A.DC. & Carycaceae & 2 \\
\hline 1060 & Jatobá & Hymenaea courbaril Linn. & Caesalpi neaceae & 3 \\
\hline 1061 & Jequetibá branco & Cariniana estrellensis (Mart.) Kuntze & Lecythidac eae & 5 \\
\hline 1062 & Jequetibá rosa & Carinia na legalis (Raddi) Kuntze & Lecythidac eae & 15 \\
\hline 1064 & Limeira & Citrus sp. & Rutaceae & 2 \\
\hline 1065 & Mamica de porca & Zanthoxylum sp. & Rutaceae & 2 \\
\hline 1066 & Mandiocão & Shefflera $\mathrm{sp}$. & Araliaceae & 1 \\
\hline 1067 & Marin heirinho & Matayba elaeagnoides Radlk. & Sapindaceae & 11 \\
\hline 1068 & Marinheiro & Guarea Kunthiana Juss. & Meliaceae & 14 \\
\hline 1069 & Monjoleiro & Acacia polyphylla DC. & Mimosaceae & 16 \\
\hline 1071 & Óleo de copaíba & Copaifera langsdorffii Desf. & Caesa lpineace ae & 4 \\
\hline 1072 & Orelha de mateiro & Chrysophyllum gonocarpum (Mart \& Eichl) Engl. & Sapotaceae & 13 \\
\hline 1073 & Orvalho & Trichillia pallens $\mathrm{C}$. & Meliaceae & 22 \\
\hline 1075 & Paineira branca & Pseudobombax grandiflorum (Carv.) A. Robins & Bombacaceae & 1 \\
\hline 1076 & Palmito & Euterpe edulis Mart. & Arecaceae & 2 \\
\hline 1077 & Panacéia & Solanum cernuum Vell. & Solanaceae & 7 \\
\hline 1079 & Pau alho & Galesia integrifolia Spreng. Harms. & Phytollacaceae & 1 \\
\hline 1080 & Pau ferro & Myrtaceae 3 & Myrtaceae & 10 \\
\hline 1082 & Pau terra & Centrolobium sp. & Fabac eae & 8 \\
\hline 1083 & Pau viola & Alchornea triplinervia Muell. Arg. & Euphorbiaceae & 10 \\
\hline 1084 & Pereira & Platyciamus regnelli Benth. & Fabac eae & 8 \\
\hline 1085 & Peroba & Aspidosperma sp. 4 & Apocynaceae & 1 \\
\hline 1087 & Peroba canela de velho & Aspidosperma sp. 2 & Apocynaceae & 6 \\
\hline 1088 & Peroba poca & Aspidosperma sp. 1 & Apocynaceae & 7 \\
\hline 1089 & Peroba rosa & Aspidosperma polyneuron Muell. Arg. & Apocynaceae & 29 \\
\hline 1091 & Pessegueiro bravo & Prunus subcoriacea Koehne. & Rosaceae & 9 \\
\hline 1092 & Pindaíba & Xylopia sp. & Annonaceae & 15 \\
\hline 1095 & Quaresma & Miconia discolor DC. & Melastomataceae & 1 \\
\hline 1096 & Quatiguá & Trichillia $\mathrm{sp}$ & Meliaceae & 36 \\
\hline 1098 & Sangueiro & Pterocarpus violaceus Vog. C19 & Fabac eae & 12 \\
\hline 1099 & Sassafrás & Ocotea pretiosa Benth. \& Hook. & Lauracae & 7 \\
\hline
\end{tabular}




\begin{tabular}{|c|c|c|c|c|}
\hline CÓDIGO & NOME VULGAR & NOME CIENTÍFICO & FAMÍLIA & $\begin{array}{c}\text { OCORRÊNCIA } \\
\text { (N }^{\circ} \text {. DE } \\
\text { PARCELAS) }\end{array}$ \\
\hline 1100 & Serralha & Sorocea guillerminiania Gaudich. & Moraceae & 15 \\
\hline 1104 & Três folhas & Esenbeckia grandiflora Mart. & Rutaceae & 37 \\
\hline 1105 & Unha de boi & Bauhinia forficata Link. & Mimosaceae & 5 \\
\hline 1108 & Veludo & Chomesia sp. & Rubiaceae & 9 \\
\hline
\end{tabular}

Nas Tabelas 3 e 4 estão apresentadas as distribuições do número total de indivíduos, número de espécies e gêneros por família botânica, respectivamente para o estrato arbóreo e para a regeneração natural.

\begin{tabular}{|c|c|c|c|c|c|c|}
\hline \multicolumn{7}{|c|}{ TABELA 3 - GÊNEROS, ESPÉCIES E NÚMERO DE INDIVÍDUOS POR FAMÍLIA P ARA A FLORESTA } \\
\hline NÚMERO & FAMÍLIA & GÊNEROS & ESPÉCIES & ESPÉCIES/GÊNEROS & FREQÜÊNNCIA & FREQÜÊNCIA (\%) \\
\hline 1 & ANACARDIACEAE & 2 & 2 & 1,00 & 430 & 6,56 \\
\hline 2 & ANNONACEAE & 3 & 5 & 1,67 & 77 & 1,18 \\
\hline 3 & APOCYNACEAE & 1 & 7 & 7,00 & 145 & 2,21 \\
\hline 4 & ARALIACEAE & 2 & 2 & 1,00 & 6 & 0,09 \\
\hline 5 & ARECACEAE & 3 & 3 & 1,00 & 54 & 0,82 \\
\hline 6 & BIGNONIACEAE & 2 & 3 & 1,50 & 62 & 0,94 \\
\hline 7 & BOMBACACEAE & 3 & 3 & 1,00 & 103 & 1,57 \\
\hline 8 & BORAGINACEAE & 1 & 3 & 3,00 & 6 & 0,09 \\
\hline 9 & BURSERACEAE & 1 & 1 & 1,00 & 2 & 0,03 \\
\hline 10 & CAESALPINEACEAE & 4 & 4 & 1,00 & 92 & 1,40 \\
\hline 11 & CARYCACEAE & 1 & 1 & 1,00 & 48 & 0,73 \\
\hline 12 & CECROPIACEAE & 1 & 1 & 1,00 & 88 & 1,34 \\
\hline 13 & CHRYSOBALANACEAE & 1 & 1 & 1,00 & 2 & 0,03 \\
\hline 14 & CLUSIACEAE & 2 & 2 & 1,00 & 286 & 4,36 \\
\hline 15 & COMBRETACEAE & 2 & 3 & 1,50 & 40 & 0,61 \\
\hline 16 & DESCONHECIDA & 1 & 1 & 1,00 & 428 & 6,53 \\
\hline 17 & ELAEOCARPACEAE & 1 & 1 & 1,00 & 1 & 0,01 \\
\hline 18 & EUPHORBIACEAE & 2 & 2 & 1,00 & 734 & 11,20 \\
\hline 19 & FABACEAE & 9 & 10 & 1,11 & 346 & 5,28 \\
\hline 20 & FLACOURTIACEAE & 1 & 2 & 2,00 & 65 & 0,99 \\
\hline 21 & LAURACEAE & 7 & 9 & 1,29 & 346 & 5,28 \\
\hline 22 & LECYTHIDACEAE & 1 & 2 & 2,00 & 212 & 3,24 \\
\hline 23 & MELASTOMATACEAE & 1 & 1 & 1,00 & 10 & 0,15 \\
\hline 24 & MELIACEAE & 4 & 7 & 1,75 & 1130 & 17,24 \\
\hline 25 & MIMOSACEAE & 7 & 8 & 1,14 & 686 & 10,46 \\
\hline 26 & MIRISTICACEAE & 1 & 1 & 1,00 & 10 & 0,15 \\
\hline 27 & MONIMIACEAE & 1 & 1 & 1,00 & 82 & 1,25 \\
\hline 28 & MORACEAE & 3 & 4 & 1,33 & 160 & 2,45 \\
\hline 29 & MYRSINACEAE & 1 & 1 & 1,00 & 12 & 0,18 \\
\hline 30 & MYRTACEAE & 6 & 6 & 1,00 & 33 & 0,50 \\
\hline 31 & NYCTAGINACEAE & 1 & 1 & 1,00 & 1 & 0,02 \\
\hline 32 & OLACACEAE & 1 & 1 & 1,00 & 3 & 0,05 \\
\hline 33 & PHYTOLLACACEAE & 1 & 1 & 1,00 & 10 & 0,15 \\
\hline 34 & PROTEACEAE & 1 & 1 & 1,00 & 21 & 0,32 \\
\hline 35 & RHAMNACEAE & 1 & 1 & 1,00 & 25 & 0,38 \\
\hline 36 & ROSACEAE & 1 & 1 & 1,00 & 26 & 0,40 \\
\hline 37 & RUBIACEAE & 5 & 5 & 1,00 & 25 & 0,39 \\
\hline 38 & RUTACEAE & 3 & 4 & 1,33 & 142 & 2,17 \\
\hline 39 & SAPINDACEAE & 3 & 3 & 1,00 & 116 & 1,77 \\
\hline 40 & SAPOTACEAE & 1 & 1 & 1,00 & 169 & 2,58 \\
\hline 41 & SOLANACEAE & 1 & 2 & 2,00 & 6 & 0,09 \\
\hline
\end{tabular}




\begin{tabular}{|c|c|c|c|c|c|c|}
\hline NÚMERO & FAMÍLIA & GÊEEROS & ESPÉCIES & ESPÉCIES/GÊNEROS & FREQÜÊENCIA & FREQÜÊNCIA (\%) \\
\hline 42 & STYRACACEAE & 1 & 2 & 2,00 & 16 & 0,24 \\
\hline 43 & ULMACEAE & 1 & 1 & 1,00 & 2 & 0,03 \\
\hline 44 & URTICACEAE & 1 & 1 & 1,00 & 291 & 4,44 \\
\hline 45 & VERBENACEAE & 1 & 1 & 1,00 & 5 & 0,08 \\
\hline \multirow[t]{2}{*}{46} & VOCHYSIACEAE & 1 & 1 & 1,00 & 1 & 0,02 \\
\hline & TOT AL & 99 & 124 & 61,62 & 6555 & 100,00 \\
\hline
\end{tabular}

\begin{tabular}{|c|c|c|c|c|c|c|}
\hline \multicolumn{7}{|c|}{$\begin{array}{r}\text { TABELA } 4 \text { - GÊNEROS, } \\
\text { NATURAL }\end{array}$} \\
\hline NÚMERO & FAMILIA & GÊENEROS & ESPÉCIES & N/HA & N/HA (\%) & ESPÉCIES/GÊNEROS \\
\hline 1 & ANACARDIACEAE & 2 & 2 & 2512,68 & 1,98 & 1,00 \\
\hline 2 & ANNONACEAE & 2 & 2 & 5830,31 & 4,58 & 1,00 \\
\hline 3 & APOCYNACEAE & 2 & 5 & 8127,15 & 6,39 & 2,50 \\
\hline 4 & ARALIACEAE & 1 & 1 & 1137,39 & 0,89 & 1,00 \\
\hline 5 & ARECACEAE & 2 & 2 & 3816,66 & 3.00 & 1,00 \\
\hline 6 & BIGNONIACEAE & 2 & 2 & 235,55 & 0,19 & 1,00 \\
\hline 7 & BOMBACACEAE & 1 & 1 & 1008,2 & 0,79 & 1,00 \\
\hline 8 & CAESALPINEACEAE & 2 & 2 & 5757,76 & 4,53 & 1,00 \\
\hline 9 & CARICACEAE & 1 & 1 & 1111,13 & 0,87 & 1,00 \\
\hline 10 & CECROPIACEAE & 1 & 1 & 200,78 & 0,16 & 1,00 \\
\hline 11 & CLUSIACEAE & 1 & 1 & 2782,51 & 2,19 & 1,00 \\
\hline 12 & COMBRETACEAE & 1 & 1 & 545,15 & 0,43 & 1,00 \\
\hline 13 & DESCONHECIDA & 1 & 1 & 3822,99 & 3,01 & 1,00 \\
\hline 14 & EUPHORBIACEAE & 2 & 2 & 8187,62 & 6,44 & 1,00 \\
\hline 15 & FABACEAE & 5 & 6 & 10262,94 & 8,07 & 1,20 \\
\hline 16 & FLACOURTIACEAE & 1 & 1 & 124,13 & 0,10 & 1,00 \\
\hline 17 & LAURACEAE & 4 & 4 & 7026,17 & 5,53 & 1,00 \\
\hline 18 & LECYTHIDACEAE & 1 & 2 & 2342,46 & 1,84 & 2,00 \\
\hline 19 & MELASTOMATACEAE & 1 & 1 & 2560,36 & 2,01 & 1,00 \\
\hline 20 & MELIACEAE & 3 & 6 & 15540,55 & 12,22 & 2,00 \\
\hline 21 & MIMOSACEAE & 6 & 7 & 15598,12 & 12,27 & 1,17 \\
\hline 22 & MONIMIACEAE & 1 & 1 & 573,37 & 0,45 & 1,00 \\
\hline 23 & MORACEAE & 1 & 1 & 2081,82 & 1,64 & 1,00 \\
\hline 24 & MYRSINACEAE & 1 & 1 & 1255,5 & 0,99 & 1,00 \\
\hline 25 & MYRTACEAE & 3 & 3 & 3953,83 & 3,11 & 1,00 \\
\hline 26 & PHYTOLLACACEAE & 1 & 1 & 182,68 & 0,14 & 1,00 \\
\hline 27 & PROTEACEAE & 1 & 1 & 1229,1 & 0,97 & 1,00 \\
\hline 28 & ROSACEAE & 1 & 1 & 438,83 & 0,35 & 1,00 \\
\hline 29 & RUBIACEAE & 1 & 1 & 1040,13 & 0,82 & 1,00 \\
\hline 30 & RUTACEAE & 3 & 4 & 12163,52 & 9,57 & 1,33 \\
\hline 31 & SAPINDACEAE & 2 & 2 & 2406,77 & 1,89 & 1,00 \\
\hline 32 & SAPOTACEAE & 1 & 1 & 946,12 & 0,74 & 1,00 \\
\hline \multirow[t]{2}{*}{33} & SOLANACEAE & 1 & 1 & 2332,08 & 1,83 & 1,00 \\
\hline & TOT AL & 59 & 69 & 38897,39 & 100,00 & 37,20 \\
\hline
\end{tabular}


A análise da composição florística da floresta, apresentada na Tabela 1 , indica a presença de 99 gêneros e 46 famílias botânicas, das quais predominam: Meliaceae (94,16 ind./ha), Euphorbiaceae (61,16 ind./ha), Mimosaceae (57,16 ind./ha), Anacardiaceae (35,83 ind./ha), Lauraceae e Fabaceae (28,33 ind./ha). Na distribuição do número total de indivíduos para a floresta, apresentada na Tabela 3, as famílias anteriormente citadas são responsáveis por mais de $50 \%$ da abundância absoluta da floresta, sendo que somente Meliaceae contribui com 17,24\% desse total. Das famílias encontradas, pode-se destacar também Fabaceae, que se constitui na mais rica da floresta, apresentando o maior número de espécies (10) e gêneros (9). A floresta apresenta em média 1,34 espécies por gênero, destacando o gênero Aspidosperma da família Apocynaceae, que apresenta o maior número de espécies (7). Destaca-se também Lauraceae, apresentando 7 gêneros e 9 espécies.

Silva (1990), em estudo realizado na Mata dos Godoy, na região de Londrina-PR, formação pertencente à Floresta Estacional Semidecidual, encontrou um total de 139 espécies, destacandose a família Meliaceae e Euphorbiaceae. Silveira (1993), estudando a estrutura vegetacional em uma toposseqüência, na mesma floresta (Mata dos Godoy), encontrou 83 espécies. De acordo com os autores acima citados, pode-se concluir que a floresta estudada apresenta uma composição florística semelhante à da região de ocorrência da Floresta Estacional Semidecidual de Londrina, principalmente quando comparada aos resultados de Silva (1990).

A regeneração natural da floresta apresenta uma composição florística diferente da floresta. Como pode ser observado na Tabela 2, na floresta estudada foram encontradas 69 espécies que apresentam indivíduos com DAP menor que $10 \mathrm{~cm}$, resultando um número médio de indivíduos por hectare igual a 39.021,95. A distribuição do número total de indivíduos da regeneração natural, apresentada na Tabela 4, indica a presença de 33 famílias botânicas e 58 gêneros, das quais predominam: Mimosaceae (15.598,12 ind./ha), Meliaceae (15.440 ind./ha), Rutaceae (12.163 ind./ ha), Fabaceae (10.262 ind./ha), Euphorbiaceae (8.187 ind./ha) e Apocynaceae (8.127 ind./ha).

No geral, as seis famílias botânicas relacionadas são responsáveis por cerca de 54,96\% do número total de indivíduos da regeneração natural, podendo ser destacadas Mimosaceae (mais rica, com 6 gêneros e 7 espécies) e Meliaceae, que juntas compõem cerca de $25 \%$ do total de indivíduos da regeneração.

A regeneração natural apresenta uma relação espécie/gênero média de 1.12, destacandose Apocynaceae, com 2 gêneros e 5 espécies. Destaca-se, também, o gênero Aspidosperma (Apocynaceae), que possui 5 espécies, e o gênero Trichillia (Meliaceae), com 3 espécies.

Com essas informações, pode-se comprovar que a composição florística da floresta difere consideravelmente da regeneração natural, principalmente em relação ao número de indivíduos por família. Esta diferença pode ser explicada pela ecologia das espécies, que ocupam o estrato inferior da floresta, devido as suas próprias características, como por exemplo, os representantes da Arecacaea, Rutaceae, entre outras. Por outro lado, existem espécies, por exemplo, o bálsamo, que possuem grande densidade na regeneração, mas apresentam baixa densidade na população adulta, mesmo sendo uma espécie com características de grande porte. Muitas espécies, como é o caso do quatiguá, três folhas e orvalho, possuem grande densidade de indivíduos na regeneração natural, mas, por características próprias, dificilmente atingem grandes portes, ocupando assim o estrato inferior da floresta.

Outra consideração deve ser feita com relação às espécies chamadas de oportunistas, como é o caso da embaúba e do capixingui, que ocupam rapidamente as aberturas naturais (clareiras) deixadas pela queda ou morte de outras árvores, e que somente se desenvolvem até o dossel em virtude destas situações.

Jardim (1985), em estudo realizado na Floresta Equatorial Úmida da Estação Experimental do INPA, encontrou 244 espécies na regeneração natural, distribuídas em 152 gêneros e 55 famílias botânicas. Segundo o autor, a composição florística da regeneração natural diferiu consideravelmente da composição florística da floresta, principalmente com relação à baixa freqüência ou ausência de algumas espécies, situação semelhante à encontrada na floresta estudada neste trabalho.

\section{Conclusões}

A floresta apresentou 124 espécies com DAP maior que $10 \mathrm{~cm}$, distribuídas em 99 gêneros 
e 46 famílias botânicas. No caso da regeneração natural, foram encontradas apenas 69 espécies arbóreas (DAP menor que $10 \mathrm{~cm}$ ), distribuídas em 58 gêneros e 33 famílias botânicas. Estes resultados indicam que a diversidade de espécies da população adulta é significativamente maior que a regeneração, representando quase o dobro do número de espécies.

Na população adulta, a família botânica mais rica é Fabaceae (9 gêneros e 10 espécies), e a mais representativa é Meliaceae, representando cerca de $17 \%$ da abundância total da floresta. Para a regeneração natural, destaca-se Mimosaceae, apresentando o maior número de indivíduos (cerca de $12 \%$ do total) e se constituindo na família mais rica, com 6 gêneros e 7 espécies.

$\mathrm{Na}$ regeneração natural, destacam-se as espécies três folhas (Esenbeckia grandiflora), canjica (Mimosaceae 1), pau viola (Alchornea triplinervia), quatiguá (Trichillia sp.) e bálsamo (Myrocarpus frondosus), que totalizaram cerca de $22 \%$ da regeneração da floresta. Pode-se dizer que a regeneração natural das principais espécies da floresta é satisfatória, com exceção do urtigão (Urera baccifera), com regeneração praticamente inexistente, e do marinheiro (Guarea kunthiana), que apresenta baixos valores de regeneração. De maneira geral, a regeneração natural apresentouse reduzida, visto que apenas 69, das 124 espécies encontradas nas populações adultas, ocorreram neste estrato da floresta, naturalmente, considerando-se a amostragem efetuada na população.

\section{Agradecimentos}

A todos que contribuiram direta ou indiretamente para a realização deste trabalho, especialmente: aos Engenheiros Florestais e amigos, Alexandre Koehler, Dennis Dosza, Fernando José Fabrowski e Sandro Dallacorte, pelos trabalhos de campo e manipulação dos dados; aos professores Carlos Roberto Sanquetta, Carlos Vellozo
Roderjan e Willian Thomaz Wendling, pela contribuição e auxílio; aos participantes dos trabalhos de campo, Sr. Antônio e Eduardo; ao Dr. Gert Hatschbach, pela identificação das espécies; ao Curso de Pós-Graduação em Engenharia Florestal da Universidade Federal do Paraná, pela oportunidade concedida; à Cris e à minha família, pelo auxílo nos momentos difíceis.

\section{Referências}

HOSOKAWA, R. T. Manejo Florestal. (Série didática). UFPR. Curitiba, 1986.

HUSCH, B.; MILLER, C. I.; BEERS, T. W. Forest mensuration. The Ronald: [s.1.] 1972.

JARDIM, S. F. C. da. Estrutura da floresta equatorial úmida da estação experimental de silvicultura tropical do INPA. Manaus, 1985. $195 \mathrm{f}$. Dissertação (Mestrado) - FUA.

LAMPRECHT, H. Ensayo sobre la estructura florística de la parte sur- oriental del Bosque Universitario "El Caimital "- Estado Barinas. Rev. For. Venez., v. 7, n. 10/11, p. 77-119, 1964.

PÉLLICO NETTO, S.; BRENA, D. A. Inventário Florestal. Curitiba, 1997. v.1.

RADAMBRASIL - Levantamento de recursos naturais. [s.l.: s.n.], 1978. v. 32.

SILVA, S. L. H. Fitossociologia arbórea da porção norte do Parque Estadual Mata dos Godoy, Londrina - PR. Curitiba, 1990. 197f. Dissertação (Mestrado) - UFPR.

SILVEIRA, M. Estudo vegetacional em uma toposseqüência no Parque Estadual “ Mata dos Godoy “, Londrina. Curitiba, 1993. 142f. Dissertação (Mestrado). - UFPR. 